\title{
Solid neuroendocrine carcinomas of the breast. Metastases or primary tumors?
}

Christiane Richter-Ehrenstein ${ }^{1}$, Juliane Arndt ${ }^{1}$, Ann-Christin Buckendahl ${ }^{2}$, Jan Eucker ${ }^{3}$, Wilko Weichert ${ }^{2}$, Atsuko Kasajima ${ }^{2}$, Achim Schneider $^{1}$, Aurelia Noske ${ }^{2,4}$

${ }^{1}$ Department of Gynecology, Interdisciplinary Breast Center, Charité University Medicine Berlin, Germany

${ }^{2}$ Institute of Pathology, Charité University Medicine Berlin, Germany

${ }^{3}$ Department of Oncology and Hematology, Charité University Medicine Berlin, Germany

${ }^{4}$ Institute of Clinical Pathology, University Hospital Zurich, Switzerland

Running title: neuroendocrine carcinomas of the breast

Keywords: breast cancer, neuroendocrine tumors, GCDFP-15, mammaglobin

Address correspondence to:

Christiane Richter-Ehrenstein MD

Department of Gynecology

Interdisciplinary Breast Center

University Medicine Berlin

Charité Platz 1

10117 Berlin

Telephone: 004930450664356

Fax: 004930450564922 


\section{Abstract}

Purpose: Neuroendocrine breast carcinomas are rare but may represent either metastatic or primary lesions. So far, clinical and preoperative histopathological examinations do not distinguish properly between a primary or metastatic breast tumor. Due to any possible consequences following an appropriate treatment, markers which may be helpful for such a distinguishment are needed.

Methods: We addressed this study in order to evaluate the immunohistochemical expression of GCDFP-15 and mammaglobin in a subset of pure neuroendocrine breast carcinomas $(n=9)$ and compared the expression profile with a cohort of non-mammary neuroendocrine tumors $(\mathrm{n}=99)$.

Results: We observed in our study that solid neuroendocrine breast carcinomas are characterized by the expression of estrogen and progesterone receptors as well as GCDFP-15 and/or mammaglobin. GCDFP-15 was expressed in 6 out of 9 cases, mammaglobin was positive in 4 out of 9 tumors.

In contrast, neuroendocrine tumors of the non-mammary cohort expressed neither GCDFP-15 nor mammaglobin.

Conclusions: We conclude that mammaglobin and GCDFP-15 as markers of epithelial breast origin may work as a new and reliable diagnostic tool to distinguish primary endocrine tumors of the breast from a metastatic neuroendocrine disease. This is of utmost importance, especially for surgical management. 


\section{Introduction}

Neuroendocrine tumors of the breast are rare and may represent either metastatic or primary lesions. They derive from neuroendocrine cells, which are present throughout the body, and arise most commonly in the bronchopulmonary system and gastrointestinal tract (small intestine $>$ appendix $>$ rectum). In 2003, the World Health Organization (WHO) classification of neuroendocrine tumors of the breast established the immunohistochemical expression of one or more markers (neuron specific enolase, chromogranin A, synaptophysin) in at least $50 \%$ of the tumor cells [1]. The existence of primary breast neuroendocrine tumors now accepted accounts for less than $2 \%$ of all primary breast cancers [1, 2]. The recognition of the presumptive origin of the neuroendocrine tumor in the breast is extremely important due to the fact that different types of clinical management are present to distinguish between a primary and a metastatic tumor. Mammography is an important diagnostic tool for the evaluation of any breast mass. Neuroendocrine tumors often appear as clear-cut circumscribed masses and are sometimes mistaken for fibroadenomas or cysts [3]. A diagnostic distinction between primary or metastatic neuroendocrine lesion by mammography or ultrasound is almost impossible. In histology, the neuroendocrine tumors typically consist of a uniform cell population with abundant eosinophilic cytoplasm and nuclei with stippled ("salt and pepper") chromatin. Conventional histomorphological evaluation does not allow an exact distinction between primary and metastatic lesions. Staining for estrogen receptor is not helpful because many studies have reported positive staining in both mammary and extramammary neuroendocrine tumors $[4,5,6,7]$. Clinically, some patients may present 
symptoms of a carcinoid syndrome. However, its absence does not rule out the possibility of its having an extramammary origin $[8,9,10]$.

The purpose of this study was to evaluate a new diagnostic tool to distinguish primary neuroendocrine tumors of the breast from a metastatic disease of other sites. To our knowledge, this is the first time that Gross cystic disease fluid protein (GCDFP-15) and mammaglobin have been used as acceptable immunohistochemical markers for epithelia of breast origin [11]. Furthermore, we compared these neuroendocrine breast carcinomas with a cohort of non-mammary neuroendocrine tumors. 


\section{Material and methods}

\section{Patients}

Our study included 9 female patients with breast cancer who underwent surgical treatment at the Department of Gynecology, Breast unit, University Hospital Charité Berlin, between 2003 and 2009. Formalin-fixed paraffin-embedded tissue from breast cancer patients with neuroendocrine differentiation were obtained from patients treated at the interdisciplinary breast unit of the Charité Berlin with institutional review board approval.

Histopathological diagnosis was performed at the Institute of Pathology, Charite University Hospital Berlin. Histological typing of the tumors was carried out according to the criteria of WHO 2003. Tumor stage was determined according to the guidelines of the UICC. Histological grading was performed following the criteria of Elston and Ellis [12]. Data of the hormone receptor and Her2 status, proliferative fraction (MIB-1) as well as immunohistochemical expression of neuroendocrine markers were taken from the archival pathology reports and re-evaluated.

To compare expression profiles of neuroendocrine tumors derived from the breast and extra-mammary sites, we included in this study a cohort of 99 patients with gastroenteropancreatic tumors of the foregut $(n=47)$ and midgut $(n=52)$, who received surgical treatment in the Charité University Hospital between 1983 and 2007. In 70 cases, tissue specimens were from the primary lesion as well as from nodal $(n=10)$ and distant metastases $(n=19)$. Neuroendocrine tumors were staged and graded according to the novel consensus proposal for gastroenteropancreatic neuroendocrine tumors [13]. 


\section{Immunohistochemistry}

Immunohistochemical analysis was performed on paraffin sections of the archival tissue blocks. For the non-mammary tumor collective, sections of tissue microarrays were used as described by Kasajima $\mathrm{A}$ et al. (publication submitted). The staining procedure was carried out using Discovery XT autostainer (Ventana, Tuscon, AZ, USA) according to the manufacturer's instructions. We used monoclonal mouse antibodies against GCDFP-15 (D6, Covance, New Jersey, USA), mammaglobin (clone 304-1A5, BioPrime, USA), and TTF1 (8G7G3/1, Zytomed, Germany). Evaluation of immunohistochemical staining was done by two pathologists independently of each other ( $A B, A N)$. 


\section{Results}

We retrospectively evaluated 9 invasive breast carcinomas with pure neuroendocrine differentiation defined by expression of at least one neuroendocrine marker in more than $50 \%$ of the tumor cells. The mean age of these patients was 64 years (range 49-82 years.). The tumor size varied from 0.6 to $5.0 \mathrm{~cm}$ in diameter whereby one case involved a bilateral tumor.

Histopathological evaluation revealed predominantly a solid growth pattern with features of an infiltrating ductal carcinoma, and in only one case an invasive lobular carcinoma. Tumor cells were sometimes characterized by a granular eosinophilic cytoplasm, round and relatively monomorphic nuclei with "salt and pepper" chromatin. Small cell features were not found. Neuroendocrine differentiation was then confirmed by immunohistochemical staining by at least one neuroendocrine marker. In addition, in three cases an accompanying situ component was observed. All breast carcinomas except one expressed estrogen and/or progesterone receptors at generally high levels. Her2/neu overexpression was found in only one carcinoma (1/9) and proliferative activity (MIB1) ranged from 1-40\% (mean 12\%). Diffuse expression of synaptophysin was observed in nearly all tumors (8/9), while chromogranin A was found in 6 out of 9 tumors. To differentiate between a primary solid neuroendocrine tumor of the breast and a metastatic neuroendocrine tumor of another site, we investigated the expression of GCDFP-15 and mammaglobin as markers of breast epithelium origin. GCDFP-15 was expressed in 6 out of 9 cases. In contrast, mammaglobin was positive in 4 out of 9 tumors. GCDFP-15 was always seen in combination with mammaglobin. Immunohistochemical findings are summarized in Table 1. Figure 1 shows the immunohistochemical expression of GCDFP-15 and mammaglobin in a neuroendocrine 
breast carcinoma. In three patients, both markers were negative. In two of these patients, no visible primary tumor was shown at other sites on the CT scan. One of these patients (No. 6) died for an unknown reason last year. One woman (No. 9) - which had initially been presented with bilateral breast cancer - was diagnosed as having a solid neuroendocrine carcinoma. Extensive staging revealed a neuroendocrine tumor of the midgut with multiple metastases (heart, lymph nodes, and breast).

In addition, we analyzed the expression of TTF-1 (thyroid transcription factor 1) which is known to be positive in the majority of primary adenocarcinomas and small cell neuroendocrine carcinomas of the lung. We did not observe any expression in the breast tumors. With regard to the comparison group of non-mammary neuroendocrine tumors, the mean age of patients with foregut tumors was 53 years, and with midgut tumors 58.2 years at the time of diagnosis. 51 patients $(51.5 \%)$ were male; there was no difference observed in gender distribution between tumor locations.

We did not find any expression of GCDFP-15, mammaglobin, or TTF-1 in immunohistochemical evaluation of non-mammary neuroendocrine tumors but all expressed synaptophysin and chromogranin A. 


\section{Discussion}

Neuroendocrine tumors of the breast are rare and may occur as metastases from a known neuroendocrine tumor, and may be considered as the first presentation of an occult metastatic neuroendocrine tumor, or as a primary breast tumor.

Our study demonstrates that solid neuroendocrine breast carcinomas are characterized by the expression of estrogen and progesterone receptors as well as GCDFP-15 and/or mammaglobin. In contrast, neuroendocrine tumors of the non-mammary cohort expressed neither GCDFP-15 nor mammaglobin.

Our data clearly support the use of these two markers: mammaglobin and GCDFP-15. Recently, Fritzsche et al. showed that in $91.8 \%$ of breast cancer cases $(n=165)$ at least one of these two markers is expressed. They demonstrated a strong correlation of GCDFP-15 and mammaglobin and showed that only very few primary breast cancers are completely negative for both markers [11]. Sapino et al. first described the use of GCDFP-15 for neuroendocrine breast tumors in 2001 [25]. In our study population of neuroendocrine breast cancers, 6 out of 9 tumors expressed one or both markers. One case in our series (No. 9) is of clear metastatic origin, in two cases there was good reason to suspect tumors having a primary mammary origin (Nos. 3 and 6). In our study, all neuroendocrine breast carcinomas did express hormone receptors, and this is in line with other reports in the literature [2, 14, 23]. Lopez-Bonet et al. reported in 7 out of 7 cases of solid neuroendocrine breast carcinomas estrogen und progesterone receptor positivity [2]. However, the receptor status alone is not helpful to differentiate between a primary or metastatic breast tumor. Previous reports have shown that endocrine tumors of other origin may also express hormone receptors. Sica et al. showed in a cohort of 42 neuroendocrine tumors of the lung 17 tumors with immunoreactivity against estrogen 
and in $70 \%$ of those cases, a positive progesterone staining [7]. There are also unpublished data of ours confirming these results to our non-mammary cohort.

In view of the existing literature, most papers present case reports $[4,5,15,16,17]$. In the review by Upalakalin et al. [18], the authors report on 59 cases of neuroendocrine tumors in the literature. Thirty-eight of these were primary neuroendocrine tumors of the breast, 9 were presented with breast metastases for the first time as a neuroendocrine tumor, and 15 patients were presented with a history of neuroendocrine tumor prior to breast metastasis. This underlines the importance of a thorough work-up for these patients.

The diagnosis in the cases reported was made preoperatively or postoperatively, but mostly following surgical intervention including mastectomy. There appear to be no distinctive mammographic or ultrasound characteristics of invasive neuroendocrine tumors of the breast [19]. Therefore, prior to surgical intervention, the terms "primary carcinoma" and "metastasis" have to be differentiated. Patients' clinical history and preoperative pathological diagnosis by needle core biopsies must be reviewed carefully. If the tumor morphology in the core biopsy is suspected to be due to neuroendocrine differentiation, further immunohistochemical stainings should be performed. Moreover, it is important to mention in the pathology report whether it is a neuroendocrine carcinoma with solid growth or a small cell carcinoma. Expression of GCDFP-15, mammaglobin and lack of TTF-1 as well as presence of an in-situ component support an origin of the breast. This is because bilateral tumor masses, lacking hormone receptors and GCDFP15 as well as mammaglobin may favor a metastatic lesion. Notably, in very rare cases a mammography or biopsy can provoke a carcinoid crisis [20]. 
There is a paucity of clear recommendations regarding surgical management of these rare tumors. It appears that patients with primary neuroendocrine carcinomas of the breast should be treated in a similar fashion to invasive ductal breast cancer which is appropriate for the size and stage of the lesion [21, 22]. Patients with neuroendocrine metastatic disease should undergo lumpectomy for debulking. Axillary node dissections are not needed, and mastectomies must be avoided. Fishman et al. reviewed that in 8 out of 13 patients with metastatic neuroendocrine tumors to the breast; these patients were initially considered to have primary breast carcinomas and were treated with mastectomy [10].

Neuroendocrine tumors from the midgut cause functional symptoms due to the secretion of various peptides and hormones and most notably 5-hydroxytryptamine $(5-\mathrm{HT})$ or serotonin. Serum measurements are non-specific for diagnosis and follow-up due to large individual variations [24].

Patients with metastases of the breast are, on the average, 10 years younger than patients with primary neuroendocrine tumors of the breast [18]. Overall survival or disease-free survival is slightly better among patients having primary tumors with neuroendocrine differentiation when compared to patients with other breast tumors [22]. Antihormonal therapy is also the standard adjuvant therapy in neuroendocrine breast cancers. In the past decade tremendous improvement in the diagnostic work-up of patients with neuroendocrine tumors was made by using new scintigraphy tools and PET-CT scans with different tracers which have to be used in order to discriminate a metastatic from a primary disease.

In conclusion, mammaglobin and GCDFP-15 as markers of epithelial breast origin may work as a new reliable diagnostic tool to distinguish endocrine tumors of the breast from 
a metastatic neuroendocrine disease. This is of utmost importance especially for surgical management. Taking into consideration the fact that this is one of the first descriptions of using mammaglobin and GCDFP-15 as markers in this context, further evaluation is warranted.

\section{Acknowledgment}

The authors of this work have not received any funding or financial support. 


\section{References}

1. WHO (2003) World Health Organization Classification of Tumours. Pathology and Genetics of Tumours of the Breast and Female Genital Organs.In: Tavassoli FA, Devilee P (eds). IARC Press, Lyon, p9-112

2. Lopez-Bonet E, Alonso-Ruano M, Barraza G, et al. Solid neuroendocrine breast carcinomas: incidence, clinico-pathological features and immunohistochemical profiling. Oncol Rep 2008;20(6):1369-74

3. Harrist TJ, Kalisher L. Breast metastasis: un unusual manifestation of a malignant carcinoid tumor. Cancer 1977;40;3102-6

4. Cubilla AL. Woodruff JM. Primary carcinoid of the breast. Am J Surg Pathol $1977 ; 1: 283-92$

5. Ni K, Bibbo M. Fine needle aspiration of mammary carcinoma with features of a carcinoid tumor. Acta Cytol 1994;38:73-8

6. Rindi G, Kloeppel G. Endocrine Tumors of the gut and pancreas tumor biology and classification. Neuroendocrinology 2004;80(suppl1):12-15

7. Sica G, Wagner P, Altorki N, Port J, et al. Immunohistochemical Expression of Estrogen and Progesterone Receptors in Primary Pulmonary Neuroendocrine 
Tumors. Arch Pathol Lab Med 2008;132:1889-1895

8. Kaltsas GA, Putignano P, Mukherjee $\mathrm{JJ}$, et al. Carcinoid tumours presenting as breast cancer; the utility of radionuclide imaging with 123 I-MIBG and 111 InDTPA pentetrotide. Clin Endocrinol 1998;49:685-9

9. Di Palma S, Andreoal S, Luciano L, et al. Ileal carcinoid metastatic of the breast. Tumorigenesis 1988;74:321-7

10. Fishman A, Kim HS, Girtanner RE, et al. Solitary breast metastasis as first manifestation of ovarian carcinoid tumor. Gyncel Oncol 1994;54:222-6

11. Fritzsche FR, Thomas A, Winzer KJ, Beyer B et al. Co-expression and prognostic value of gross cystic disease fluid protein 15 and mammaglobin in primary breast cancer. Histol Histopathol 2007;22:1221-1230

12. Elston CW, Ellis IO. Pathological prognostic factors in breast cancer: I. The value of histologic grade in breast cancer: experience from a large study with long-term follow-up. Histopathology 1991;19:403-10

13. Rindi G, Kloppel G, Ahlmann H, et al. TNM staging of foregut (neuro) endocrine tumors: a consensus proposal including a grading system. Virchows Arch 2006;449:395-401 
14. Monsunjac M, Kochhar R, Monsunjac MI, Lau S. Primary small bowel carcinoid tumor with bilateral breast metastases. Report of 2 Cases with different clinical presentations. Arch Path Lab Med 2004;128:292-297

15. Hartgrink HH, Lagaay MB, Spaander PJ, et al. A series of carcinoid tumours of the breast. Eur J Surg Oncol 1995;21:609-12

16. Taxy JB, Tischler AS, Insalaco SJ, et al. „Carcinoid“ tumor of the breast. Hum Pathol 1981;12:170-9

17. Fisher ER, Palekar AS, Collaborators N. Solid and mucinous varieties of so-called mammary carcinoid tumors. Am J Clin Path 1979;72:909-16

18. Upalakalin JN, Collins L, Tawa N, Parangi S. Carcinoid tumors in the breast. Am J Surg 2006;191:799-805

19. Adams RF, Parulekar V, Hughes C, Kadour MJ, Talbot D. Radiologic characteristics and management of screen-detected metastastic carcinoid tumor of the breast: a case report. Clin Breast Cancer 2009;9(3):189-92

20. Ozgen A, Demirkazik FB, Arat A, et al. Carcinoid crisis provoked by mammographic compression of metastatic carcinoid tumour of the breast. Clin Radiol 2001;56:250-1 
21. Jablon LK, Somers RG, Kim PY. Carcinoid tumor of the breast: treatment with breast conservation in three patients. Ann Surg Oncol 1998;5:261-4

22. Makretsov N, Gilks CB, Coldman AJ, et al. Tissue microarray analysis of neuroendocrine differentiation and ist prognostic significance in breast cancer. Hum Pathol 2003;34:1001-1008

23. David O, Bhattacharjee M. Diffuse neuroendocrine differentiation in a morphologically composite mammary infiltrating ductal carcinoma: a case report and review of the literature. Arch Pathol Lab Med 2003;127:e131-e134

24. O'Toole D, Grossman A, Gross D, et al. ENETS Consensus Guidelines for the Standards of Care in Neuroendocrine Tumors: Biochemical Markers. Neuroendocrinology 2009; 90:194-202

25. Sapino A, Righi L, Cassoni P et al. Expression of Apocrine Differentiation Markers in Neuroendocrine Breast Carcinomas of Aged Women. Mod Pathol 2001;14:768776 
Table 1 Immunohistochemical expression profiles of neuroendocrine breast carcinomas

\begin{tabular}{|c|c|c|c|c|c|c|c|c|c|}
\hline $\begin{array}{c}\text { Patient } \\
\text { No. }\end{array}$ & $\begin{array}{l}\text { Estrogen } \\
\text { receptor }\end{array}$ & $\begin{array}{l}\text { Progesterone } \\
\text { receptor }\end{array}$ & $\begin{array}{c}\text { Her2/ } \\
\text { neu }\end{array}$ & MIB 1 & $\begin{array}{c}\text { Synapto- } \\
\text { physin }\end{array}$ & $\begin{array}{l}\text { Chromo- } \\
\text { granin A }\end{array}$ & $\begin{array}{l}\text { Mamma- } \\
\text { globin }\end{array}$ & $\begin{array}{c}\text { GCDFP- } \\
15\end{array}$ & TTF-1 \\
\hline 1 & $100 \%$ & $100 \%$ & 0 & $5-8 \%$ & pos & pos & pos & pos & $x$ \\
\hline 2 & $80 \%$ & $80 \%$ & 0 & $15-20 \%$ & pos & pos & pos & neg & neg \\
\hline 3 & $90 \%$ & $90 \%$ & $3+$ & $40 \%$ & pos & neg & neg & neg & neg \\
\hline 4 & $80 \%$ & $10 \%$ & 0 & $1-2 \%$ & neg & pos & pos & pos & neg \\
\hline 5 & $80 \%$ & $60 \%$ & 0 & $x$ & pos & pos & pos & pos & neg \\
\hline 6 & $80 \%$ & $50 \%$ & $1+$ & $x$ & pos & pos & neg & neg & neg \\
\hline 7 & $80 \%$ & $80 \%$ & 0 & $5 \%$ & pos & neg & pos & pos & neg \\
\hline 8 & $80 \%$ & $0 \%$ & 0 & $5 \%$ & pos & neg & pos & neg & neg \\
\hline 9 & $0 \%$ & $0 \%$ & 0 & $x$ & pos & pos & neg & neg & neg \\
\hline
\end{tabular}




\section{Figure 1}

Solid (A) and trabecular (B) growth in a neuroendocrine carcinoma of the breast. More than $50 \%$ of the tumor cells show immunoreactivity for synaptophysin (C) and chromogranin (D). Several tumor cells are strongly positive for mammaglobin (E) and show a moderate cytoplasmic expression for GCDFP-15 (F).

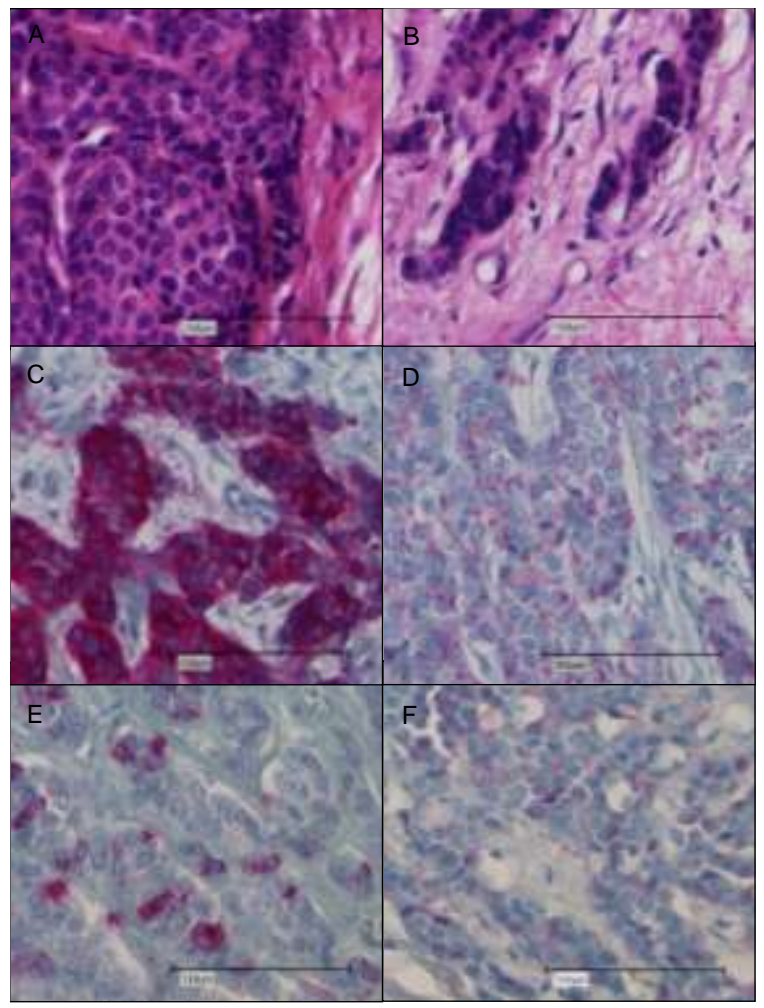

